Ebisu $\quad$ Ebisu

Études japonaises Études japonaises

55 | 2018

La fabrique des villes japonaises contemporaines

\title{
FUKUZAWA Yukichi, L'appel à l'étude, traduit, annoté et présenté par Christian Galan
}

Paris, Les Belles Lettres, 2018, 218 p.

Pierre-François Souyri

\section{(2) OpenEdition}

Journals

Édition électronique

URL : http://journals.openedition.org/ebisu/3210

DOI : 10.4000/ebisu.3210

ISSN : 2189-1893

Éditeur

Institut français de recherche sur le Japon à la Maison franco-japonaise (UMIFRE 19 MEAE-CNRS)

Édition imprimée

Date de publication : 15 décembre 2018

Pagination : 254-260

ISSN : $1340-3656$

Référence électronique

Pierre-François Souyri, « FukuzaWa Yukichi, L'appel à l'étude, traduit, annoté et présenté par Christian Galan », Ebisu [En ligne], 55 | 2018, mis en ligne le 15 décembre 2018, consulté le 10 décembre 2020 URL : http://journals.openedition.org/ebisu/3210 ; DOI : https://doi.org/10.4000/ebisu.3210 
acculturations, aux intermédiaires et médiateurs, en rejoignant ainsi des courants déjà bien établis en France et ailleurs. Mais cette histoire est aussi replacée dans le contexte de la reconfiguration des équilibres régionaux dans l'océan Indien et le golfe Persique, et surtout en mer de Chine, avec la relève des dynasties Ming et Qing, et le rétablissement à cette époque d'une politique stricte des échanges maritimes et diplomatiques. Surtout, l'évolution des compagnies des Indes à partir des modèles nationaux d'associations marchandes, vers des structures de plus en plus dominées par les intérêts politiques des États européens, le conduit vers une histoire de l'évolution des impérialismes qui se clôt à la veille de l'avènement des États-nations et la fin de l'ordre international qui avait stabilisé les mers d'Asie des temps modernes. La synthèse historique est donc à la fois synthèse méthodologique, entre plusieurs domaines de l'histoire mondiale (économique, politique et impériale, culturelle, etc.), mais aussi synthèse des traditions historiographiques européennes et nord-américaine, auxquelles viennent répondre les résultats de la recherche sur les différents peuples, États et empires qui bordaient ces mers d'Asie. Haneda nous donne ainsi une démonstration convaincante des vertus de la sortie des ghettos académiques pour s'ouvrir au monde, en invitant, plutôt qu'à le contempler de sa fenêtre pour finir par voyager autour de sa chambre, à vraiment se confronter à l'histoire écrite par les «autres».

$$
\begin{array}{r}
\text { Guillaume CARRÉ } \\
\text { Maître de conférences HDR } \\
\text { à l'EHESS }
\end{array}
$$

() Fukuzawa Yukichi

L'appel à l'étude, traduit, annoté et présenté par Christian Galan, Paris, Les Belles Lettres, 2018, 218 p.

Ce livre est d'abord un bel objet. Une couverture qui donne l'impression d'avoir l'ouvrage original entre les mains. Une reproduction intégrale $\mathrm{du}$ texte japonais du premier livre dans son édition de 1873 , qui nous fait entrer dans l'univers de l'écriture de Fukuzawa. Une photo peu connue de l'auteur, datant de 1876. Une traduction agréable, une présentation très éclairante, des notes utiles et un très beau travail d'édition. Bref, de la belle ouvrage!

Rédigé entre 1872 et 1876, L'appel à l'étude est considéré comme l'un des livres les plus importants de Fukuzawa Yukichi, et il connut 
un succès de librairie incontestable (220000 exemplaires, nous dit Christian Galan). Et, dans les écoles des années 1880, L'appel à l'étude était couramment utilisé comme manuel de classe. Comme souvent dans l'édition japonaise, on trouve à l'origine un chapitre, puis l'auteur, poussé par ses proches ou par le succès, en écrit un deuxième puis un troisième, etc. La conférence donnée à Nakatsu fin 1871 fut publiée l'année suivante. Le texte était si fort et il bénéficia d'une telle réception qu'il devint le premier chapitre d'une série qui devait en compter finalement dix-sept. Ce premier «livre» (il ne compte que six pages dans l'édition française) est le plus connu et, un siècle et demi après sa publication, reste de loin plus percutant que les seize autres dans lesquels on peut repérer, il est vrai, de nombreuses redites. Comme si l'auteur, dans son travail de pédagogue, avait besoin de dire et répéter avec d'autres mots et d'autres exemples sa philosophie première.

Le premier livre de L'appel à l'étude fut rédigé juste après la décision du nouveau gouvernement Meiji de supprimer les fiefs féodaux. Décision radicale et fondamentale qui mit un terme définitif à l'ancien régime, et laissa de côté les interprétations qui prévalaient alors, à savoir que les fiefs du sud-ouest vainqueurs des Tokugawa allaient sans doute créer un nouveau shogunat. C'est de ce moment que date le ralliement de Fukuzawa, et de la plupart des intellectuels qui se regrouperont en 1873 dans la Société de l'An VI, à un régime dont ils se méfiaient. Comme l'écrit Christian Galan dans sa préface, la situation est alors "flottante" et le pays se trouve face à une grande incertitude : impossible de se référer à un modèle préexistant. Que faire? Il faut donc inventer quelque chose de neuf et le Japon va en effet se lancer dès lors dans une expérience originale et complètement nouvelle dont Fukuzawa sera sans conteste l'un des principaux penseurs.

Lidée centrale que Fukuzawa reprend d'ouvrages occidentaux divers sans jamais les citer (Thomas Buckle et François Guizot pour l'essentiel), c'est que développer le pays pour l'enrichir et renforcer sa puissance militaire (fukoku kyōhei 富国 強兵) afin de résister à toute tentative d'agression occidentale est nécessaire mais insuffisant. Il faut aussi faire naître des hommes civilisés, c'està-dire modernes, des Japonais qui pensent différemment. Pour cela, il faut les éduquer, élever leur niveau de conscience, en faire des individus autonomes. Fukuzawa comprend la civilisation comme processus accumulatif du savoir de tous les individus qui composent la nation. Non seulement il faut introduire de nouveaux 
modes de pensée dans les systèmes, les institutions, les démarches scientifiques, mais il faut faire évoluer les mentalités et les attitudes des Japonais vis-à-vis des connaissances nouvelles.

Loriginalité de Fukuzawa, c'est qu'il invoque à la fois la nécessité de l'autonomie des individus face à l'État (ce qui sera à la base du futur mouvement pour les droits du peuple, minken 民権) et la nécessité de l'indépendance de l'État face aux grandes puissances (kokken 国権). Son Appel à l'étude repose sur deux idées. D'abord, l'égalité entre les hommes qu'il comprend surtout comme une autonomie de l'individu; il ouvre d'ailleurs son essai par une formule restée célèbre : «Le Ciel ne crée aucun homme supérieur ni aucun homme inférieur aux autres hommes. Cela signifie qu'étant tous engendrés par le Ciel, les hommes sont égaux entre eux et qu'il n'existe pas à la naissance de distinctions de rang ou de classe» (p. 47). La formule de Fukuzawa est une adaptation de la Déclaration d'Indépendance américaine qui commence par All men are created equal, mais qu'il ne cite pas non plus. Cette phrase qui bouleversa nombre de lecteurs fut lue, recopiée, commentée à l'infini dans les années qui suivirent. Personne n'avait jamais affirmé avec autant de vigueur le principe de l'égalité fondamentale des êtres humains, à commencer par les nouveaux dirigeants du pays qui se gardaient bien d'aller si loin.

Par ailleurs, Fukuzawa évoque le principe de l'indépendance comprise comme une égalité entre pays. "La liberté et l'indépendance concernent autant les nations que les individus" (p. 49). Et il unit les deux principes. Son objectif, c'est la naissance de personnes autonomes mues par une énergie elle-même produit de leur éducation, et qui construisent une nation civilisée et autonome, capable de faire face aux défis extérieurs : «ne pas craindre d'avoir à affronter les bateaux de guerre anglais et américains» (p. 49).

Indépendance nationale mais aussi de la personne, droits de l'individu et liberté de pensée sont des concepts qui traversent cette œuvre. En proclamant, dès le début, que le peuple devait s'émanciper et se rendre indépendant, Fukuzawa met le doigt sur une des faiblesses du nouveau pouvoir. Dans son Appel à l'étude, il insiste sur «l'indépendance de la personne», d'où l'importance de consacrer des forces à l'apprentissage, l'éducation pour faire apparaitre les individus autonomes et les talents dont le pays a besoin. Le Japon doit être conscient face au monde entier qu'un haut niveau d'éducation populaire est le meilleur garant de l'indépendance nationale. Tous les pays sont égaux entre eux mais, quand il 
n'existe pas dans le peuple d'un pays donné cette volonté d'indépendance, nous dit en substance Fukuzawa, il devient impossible pour ledit pays de prétendre faire respecter ses droits.

La passion de Fukuzawa Yukichi pour l'indépendance, et la nécessaire réforme des esprits qu'elle implique, se développe chez lui en discours sur l'édification d'une société de classes moyennes cultivées capables de construire la nation émancipée qu'il appelle de ses vœux. Chaque individu doit donc s'élever personnellement pour que la nation entière s'élève à son tour, sous la conduite d'une élite lettrée. Plus encore que la morale, le savoir est essentiel. Pourquoi l'Occident est-il parvenu à s'élever? Parce que les Occidentaux ont pu formuler différentes philosophies et des opinions diverses qui ont pu coexister. Se rendre autonome vis-à-vis des différents pouvoirs pour l'individu, c'est en fin de compte permettre à la nation elle-même de progresser dans la voie de la civilisation, c'est-àdire de l'indépendance. Et, pour être indépendant, le Japon doit être aussi "civilisé» que l'Occident. La civilisation, c'est l'étude. "Un homme qui n'étudie pas est un ignorant et un homme ignorant est un imbécile» (p. 47). Ou encore : "L'ignorance extrême conduit à la disparition de tout sentiment de honte» (p. 51).

Mais on peut aussi noter clairement ce que les marxistes appelleraient un «discours de classe» vis-à-vis d'un peuple que Fukuzawa méprise finalement, et ceci se retrouve dans un certain nombre de phrases où le mot “peuple» (banmin 萬民) est associé aux termes ignorance, débauche, stupidité, paresse, faiblesse, vulgarité, servilité. "Ceux qui sombrent dans la pauvreté et sont tenaillés par le froid et la faim oublient qu'ils en sont là par leur propre faute et s'en prennent fort injustement aux gens riches qui les entourent, allant, en dernière extrémité, jusqu'à former des factions (tōo musubu 党を結ぶ) et à user de violence dans des protestations en bande ou en insurrection (göto ikki 強訴一揆)» (p. 51). On perçoit bien l'allusion aux si nombreuses révoltes populaires qui, au moment même où il écrit, continuent de secouer les provinces du pays. Mais dire que la misère populaire dans le Japon féodal est de "la faute» du peuple revient à justifier le système qu'il combat. Pourtant, plus loin, il évoque les guerriers qui volent les paysans (p. 144). Pour lui le «despotisme» - et il entend par là le pouvoir seigneurial dans une société de nature féodale - n'est rien d'autre que le produit de la stupidité du peuple. Le peuple est toujours «le vrai responsable du malheur qui le frappe" (p. 58). Cette manière de voir les choses marque les limites du discours 
libéral de Fukuzawa dont Christian Galan rappelle dans sa postface à quel point il s'inspire du discours conservateur dominant au XIX ${ }^{e}$ siècle. Quand «le peuple» cherche à prendre en main son destin dans les années 1880 avec le mouvement pour la liberté et les droits du peuple en pétitionnant pour l'obtention d'une constitution, Fukuzawa, qui est resté sur le chemin de l'histoire, commente avec mépris : «Ils demandent une constitution mais savent-ils seulement ce qu'est une constitution ${ }^{1}$ ?». C'est que l'on ne se dégage pas si facilement du complexe de supériorité qui anime en permanence Fukuzawa, lui qui, par son œuvre, se pose toujours en penseur de l'avant-garde intellectuelle, critiquant les lettrés traditionnels : il traite tel propos de Mencius de stupide (p. 102) ou les enseignements de Confucius de désuets (p. 136), il se moque des moines zen (qui sont «si détachés du monde qu'ils n'ont en réalité aucune opinion sur rien", p. 127), des savants hollandistes (qui sont en vérité des fainéants qui se contentent de «lire des livres mais jamais de faire travailler leur esprit", p. 126). Car il y a bien chez Fukuzawa Yukichi un côté donneur de leçon, qui finit par être lassant...

Quelques mots maintenant sur la traduction très fluide. On ne saurait trop remercier ici le traducteur de s'être plongé dans un texte qui constitue un classique de la pensée japonaise de la fin du XIX ${ }^{e}$ siècle, écrit dans une langue difficile pour celui qui ne serait familier que du japonais contemporain. Les critiques que je porte ici sont amicales et je me permets de les signaler d'autant plus aisément que j'ai moi-même commis dans d'autres publications des erreurs de même nature. Les problèmes sont en grande partie liés au fait que la langue japonaise hésite encore dans ces années-là car les concepts du vocabulaire politique ne sont pas encore fixés. Il y a une fluctuation des mots qui rend le travail du traducteur encore plus complexe.

Christian Galan traduit le mot banmin (qui apparaît très fréquemment chez Fukuzawa) par peuple et il a sans doute raison. Aujourd'hui, le mot est quelque peu désuet et on dirait vraisemblablement plus facilement minshū 民衆, voire jinmin 人民. Fukuzawa utilise parfois le terme kokumin 国民, qui possède en japonais contemporain deux significations selon le contexte : il peut désigner la population d'un pays, ou bien la nation. Le sens de kokumin comme nation s'est lui-même sans doute fixé à la fin des années 1880; en 1874, le terme est vague et peut signifier également peuple. C'est le choix de traduction que fait Christian Galan. Or, je ne pense pas que Fukuzawa utilise les deux mots banmin et kokumin 
comme synonymes, notamment dans une phrase qui fut relevée en ce tempslà parce que percutante (p. 70 dans le livre IV) : "le Japon a bien à présent un gouvernement, il n'a en revanche toujours pas de peuple». Elle vient après un passage consacré aux savants hollandistes dont Fukuzawa nous dit qu'ils «n'ont jamais fait l'expérience de ce qu'est une société fondée sur les droits civiques" (minken). Écrire dans ces conditions que le pays n'a pas de peuple n'a pas grand sens. La bonne traduction serait sans doute : "Le Japon a bien un gouvernement mais pas de nation"

À plusieurs reprises, le traducteur évoque dans le texte la «restauration Meiji». Fukuzawa n'utilise jamais cette expression qui pourrait correspondre en japonais à ösei fukko 王政古, «restauration de la monarchie». Fukuzawa écrit ōsei ichido shin nari 王制一度新なり, «le système monarchique s'est rénové d'un coup" (Livre I, p. 50). On peut comprendre que pour des raisons de fluidité, on décide de traduire autrement et de considérer qu'il s'agit de l'équivalent de Meiji ishin 明治維新. Le problème, c'est que Meiji ishin ne signifie pas la «restauration» Meiji, même si cette traduction est courante. C'est un terme qui désigne bien plus qu'une restauration, il évoque en chinois un changement révolutionnaire, lié à un changement dynastique. Ishin est l'équivalent de $k a k u m e i$ 革命, qui signifie en chinois comme en japonais contemporain "révolution", mais qui désignait à l'origine le changement de mandat du Ciel. Meiji ishin ne signifie pas la restauration Meiji mais la révolution Meiji (ou, à la rigueur, la rénovation Meiji). Réduire l'ensemble des changements qui eurent lieu alors à l'événement que fut le rétablissement de l'empereur dans ses pouvoirs après la chute du bakufu est par trop réducteur, et satisfait une historiographie impériale qui a placé l'empereur au centre du discours sur la nation. Fukuzawa évoque ici les changements survenus alors (relations avec les pays étrangers, respect des lois internationales), et il dit que cette rénovation a montré «au peuple (jinmin) le vrai sens de la liberté et de l'indépendance», en la qualifiant d' «un des événements les plus importants de notre histoire». Nulle part, il n'évoque luimême la restauration monarchique. D'ailleurs le mot empereur n'apparaît nulle part, sauf erreur, dans l'ensemble de L'appel à l'étude.

Dernier point, l'expression bunmei $k a i k a$ 文明開化, traduite par «civilisation et ouverture» par Christian Galan (livre VIII p. 99). Bunmei signifie en effet civilisation mais kaika aussi. Kaika ne signifie ni l'ouverture ni les Lumières, mais la civilisation comprise comme un processus $(k a$ 
化). À la fin du XIx $x^{e}$ siècle, on trouve fréquemment les expressions bunmei, kaika et bunmei kaika, qui sont utilisées de manière synonyme. Le kanji kai 開 renvoie à l'idée d'ouverture? Kaika signifie bunka ga hirakeru 文化 が開ける, faire progresser la culture, civiliser.

Je me permets ici de mettre ces questions de détail sur la table parce que la plupart des chercheurs occidentaux n'y sont pas attentifs, et qu'il devient urgent d'ajuster les traductions dans la mesure du possible.

Ceci dit, il faut bien entendu rendre grâce à Christian Galan pour le superbe travail effectué. L'ouvrage fera date comme source incontournable dans l'étude en français du processus de modernisation du Japon.

Pierre-François Souyri Professeur honoraire à l'université de Genève

1. Citation exacte dans Pierre-François Souyri, Moderne sans être occidental, Aux origines du Japon d'aujourd'hui, Paris, Gallimard, 2016, p. 163.

2. Watanabe Hiroshi, $A$ History of Japanese Political Thought 1600-1901, David Noble (trad.), Tokyo, International House of Japan, 2012, p. xiv.
(2) Karatani Kōjin 柄谷行人 Tetsugaku no kigen 哲学の起源 (Les origines de la philosophie) Tokyo, Iwanami shoten 岩波書店, 2012, $246 \mathrm{p}$.

Traduction en anglais par J. A. Murphy: Isonomia and the Origins of Philosophy, Durham, Duke University Press, 2017, 165 p.

Les origines de la philosophie de Karatani Kōjin n'est pas seulement une tentative de réévaluer l'apport des présocratiques au détriment de la tradition platonicienne et aristotélicienne, c'est aussi une forme de défi aux études asiatiques ${ }^{1}$. Qu'est-ce qu'un spécialiste de l'Asie peut avoir à dire d'un ouvrage japonais sur la Grèce antique? Et à l'inverse : n'y a-til pas dans ce texte des éléments susceptibles d'orienter la construction même de nos objets de recherche?

Dans ce livre, Karatani examine chapitre par chapitre les principaux auteurs de la tradition ionienne, d'Hippocrate à Empédocle, en passant par Thalès, Hérodote, Héraclite et Parménide. Il y insiste à chaque fois sur le fait que leur pensée ne se résume pas à une philosophia naturalis, mais comporte, contrairement à ce qu'en a retenu la postérité, une 